\title{
Control of shell pigmentation by secretory tubules in the abalone mantle
}

\author{
Alyssa Budd ${ }^{1 \dagger}$, Carmel McDougall ${ }^{1 \dagger}$, Kathryn Green $^{2}$ and Bernard M Degnan ${ }^{{ }^{*}}$
}

\begin{abstract}
Background: Molluscan shells exhibit a wide diversity of pigmentation patterns and are often used as models for understanding the mechanisms underlying biological pattern formation. Numerous mathematical models have been put forward to describe these patterns, but all rely on assumptions regarding the nature of the pigments and the cells from which they are secreted. We investigated pigmentation and cellular morphology of the mantle (shell-secreting organ) of the tropical abalone, Haliotis asinina, as a crucial step towards understanding the molecular mechanisms of shell patterning in this gastropod mollusc.

Results: Accumulation and release of pigmented products occurs within secretory tubules in the prismatic zone of the juvenile $H$. asinina mantle. The colour observed within these tubules closely matches the colour deposited most recently within the shell lying directly above. The pigments are autofluorescent, and confocal microscopy reveals that multiple pigments can be present within a single tubule at any one time. Examination of mantle morphology in other gastropods reveals that mantle tubules are not restricted to abalone, but are not universal.

Conclusions: The presence of a tubule-based secretory system within the abalone mantle demonstrates that pigmentation is not controlled by a simple line of cells. Instead, co-ordination of patterning events is likely to be modular, with signals received by individual cells being transmitted throughout the entire tubule to synchronise the accumulation and secretion of pigmented material.
\end{abstract}

Keywords: Mollusc, Mantle, Tubule, Pigmentation, Abalone, Shell

\section{Introduction}

The shells of molluscs represent a unique example of the emergence of complex patterns during the ontogeny of an organism in which a complete record of developmental processes is preserved. This tractability led to mollusc shell pigmentation being used as one of the first models to explore the potential roles of reaction-diffusion mechanisms in morphogenesis [1]. Although such mechanisms have since been shown to perform key roles during the development of various model species such as Drosophila, chicken, and zebrafish [2], interpretation of the mechanisms underlying molluscan shell pigmentation is mostly limited to mathematical modeling of hypothetical signaling events [3-9]. These models assume that pigmentation is controlled by localised excitation and lateral inhibition

\footnotetext{
* Correspondence: b.degnan@uq.edu.au

'Equal contributors

${ }^{1}$ Centre for Marine Science, School of Biological Sciences, The University of Queensland, Brisbane, QLD 4072, Australia

Full list of author information is available at the end of the article
}

operating along a 'line' of cells (the mantle edge), however it is not known whether the cells involved in pigment secretion are organized in this manner. Likewise, the identity of the molecules involved in molluscan pigmentation and the interactions between them are unknown.

Underlying the vast phenotypic diversity of mollusc shells is an evolutionarily homologous organ - the mantle. The mantle sits in direct contact with the shell and is thought to be regionalised, in that discrete territories are responsible for the secretion of structurally distinct shell layers [10-13]. In many molluscs, these consist of a thin outer proteinaceous layer, the periostracum, which overlies one or more consecutive layers of calcium carbonate polymorphs. In bivalves and gastropods, the periostracum is secreted from within an involution of the mantle, termed the 'periostracal groove', while the other shell layers are secreted from successive regions of the upper (dorsal) mantle epithelium [14]. Molluscs typically lengthen their shells via the addition of new material at a single growing edge and it is here that new pigmentation patterns are 
established in either the periostracum or within the calcified layers themselves [15-18].

An emerging model for the study of the molecular basis of molluscan pigmentation is the juvenile of the tropical abalone, Haliotis asinina. The juvenile shell of this species exhibits a number of pattern elements which include a line of respiratory holes (tremata), alternating red and white patches, and longitudinal raised ridges displaying orange and blue spots when the background is red, and orange spots only when the background is white (Figure 1A). These pattern elements are deposited in a predictable manner at the growing shell edge. The shell is composed of an outer prismatic layer and an inner nacreous layer (Figure 1B), and is secreted by the underlying mantle, which is divided into two lobes (Figure 1C). EST datasets from the juvenile and adult mantles of $H$. asinina have been sequenced [19,20], and proteomic analysis has identified a number of proteins that are incorporated into the adult shell [21]. H. asinina can be reared in large numbers [22,23], and is the only mollusc to date for which the spatial expression of a gene has been shown to correlate precisely with shell pattern elements [19].

Previous morphological studies on the mantle ultrastructure of various abalone species have reported the presence of pigments in tubular structures found in the region of the mantle thought to give rise to the prismatic layer $[12,24]$. Similar tubular structures have been found in the mantle of juvenile $H$. asinina [10]. It has also been reported that, at least for land snails, pigmentation patterns in the mantle match those found upon the shell [25]. These findings hint that shell pigmentation may not be controlled by the simple 'line of cells' that is assumed in the mathematical models, and that the system controlling pigmentation may be significantly more complex. In this paper, we investigate the cellular basis for control of pigmentation patterns in $H$. asinina, and extend these findings to other gastropod species.

\section{Results and discussion}

\section{The prismatic zone of the abalone mantle contains} numerous tubules

Toluidine blue stained cross sections of the mantle of juvenile $H$. asinina reveal numerous invaginations of the epithelium that extend into the connective tissue of the mantle (Figure 2A, arrows, [10]). These invaginations are restricted to a particular zone of the mantle responsible for giving rise to the prismatic layer. This is evidenced by the expression of the gene Sometsuke in this region, and the identification of the Sometsuke protein in the prismatic shell layer $[19,21]$, however we note the possibility that elements of the prismatic layer may also be secreted from the periostracal groove [26]. Transmission electron
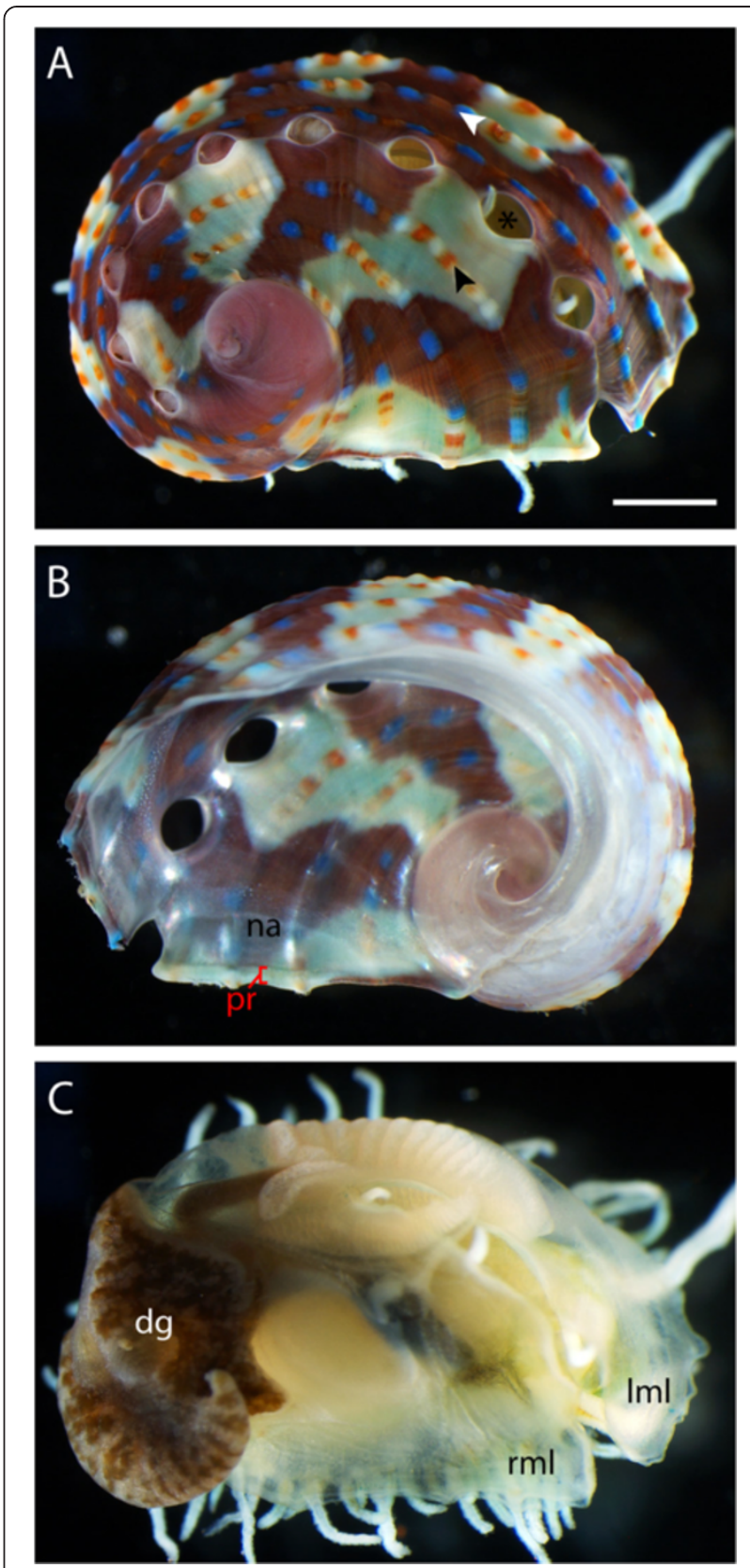

Figure 1 The shell and mantle of juvenile $\boldsymbol{H}$. asinina. A. Dorsal view of shell, anterior to the right. Tremata are indicated by an asterisk. The white arrowhead points to a ridge with orange and blue spots in an area of red background, the black arrowhead points to a ridge on a white background possessing orange spots only. Scale bar $=1 \mathrm{~mm}$.

B. Inner surface of the shell depicted in A, anterior to the left. At the shell margin, only the prismatic layer (pr) is visible, whereas the nacreous layer (na) is deposited more centrally. $\mathbf{C}$. Dorsal surface of the animal dissected from the shell, anterior to the right. The mantle is divided into two parts, the right mantle lobe $(\mathrm{rml})$ and the left mantle lobe $(\mathrm{lml})$. The digestive gland (dg) is normally situated within the whorl of the shell.

microscopy (TEM) of the tubules shows that a single layer of epithelial cells line a central canal to form a blind-ending tube that opens to the dorsal (shell- 


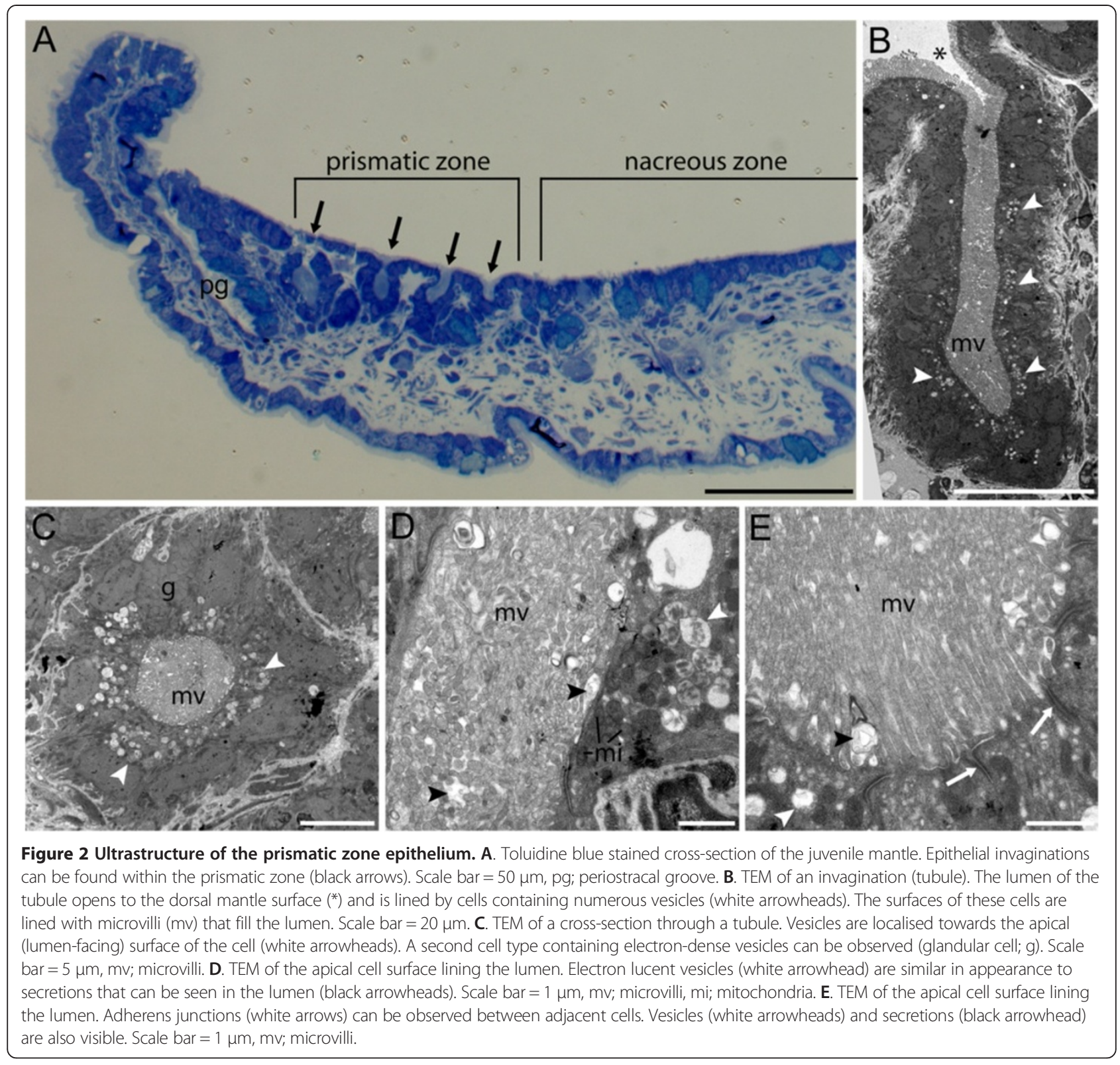

abutting) surface (Figure 2B). These cells house numerous vesicles, concentrated towards the apical (canal-bordering) microvillar surface (Figure 2B,C). Differing amounts of secreted material can be observed in the canal lumen of individual tubules (Figure 2D, see supplementary movie in Additional file 1). Due to the structure and secretory nature of these invaginations, this region of the mantle can be described as a tubular exocrine gland [27]. Some tubules were seen to merge before opening to the mantle surface (supplementary movie in Additional file 1).

The tubules are primarily comprised of one cell type, characterised by the presence of numerous electron-lucent vesicles (Figure 2B,C,D). It is likely that these vesicles are the source of the secreted material found within the lumen, given the similarity in appearance and the number of vesicles present (Figure 2D,E). The cells are connected apically via adherens junctions (Figure 2E). Occasionally, a second cell type with homogenous, electron-dense vesicles can be observed within the tubule (Figure $2 \mathrm{C}$ ). These are similar in appearance to the glandular cells described in [10] which can also be found within the non-tubular mantle epithelium of the nacreous zone.

\section{Localisation of pigment within the tubules maps to shell pigment patterns}

The tubules contain pigmented granules that can be clearly visualised using light and confocal microscopy. The colour of the pigment in the mantle corresponds (in 
most cases) with the most recent colour that has been deposited in the shell, and areas with very little visible pigment correspond to white shell patches (Figure 3A and $\mathrm{B}$, see in Additional file 2: Figure S1 for other examples). Where congruence between colours is not observed, it is likely that the mantle is accumulating pigment for the next phase of deposition (e.g., blue pigment in the boxed area in Figure 3B, given the repetitive pattern the corresponding region of the shell, will likely gain a blue spot in the near future). Confocal microscopy reveals that the pigments found within the tubules are autofluorescent, emitting light in the green, red and far-red wavelengths (Figure 3C). Higher magnification of the mantle reveals the presence of red, orange and blue pigment (Figure 3D), and the fluorescent properties of these different pigments; excitation at $433 \mathrm{~nm}$ results in green autofluoresence (Figure 3E), likely from the orange pigment, and excitation at $543 \mathrm{~nm}$ and $633 \mathrm{~nm}$ results in red and far-red autofluoresence (Figure 3F and 3G), both likely to be from the blue pigment. Fluorescence of the red pigment was not observed when excited by any of the three laser lines (arrows in Figure 3D-G). In some cases, more than one pigment can be seen within a single tubule, indicating that the tubules do not exclusively secrete one pigment type (Figure 3D-G).

Visualisation of actin filaments in the mantle by phallacidin staining reveals that muscle fibres are predominately oriented perpendicular to the mantle edge (Figure 4A and $\mathrm{C}$ ). This suggests that the mantle can be retracted away from the shell edge, but, when relaxed, sits directly flush with it. The paucity of longitudinal musculature indicates little capacity for sideways movement of the mantle, thus the pigmented tubules probably remain in alignment with the same area of the shell edge. Active

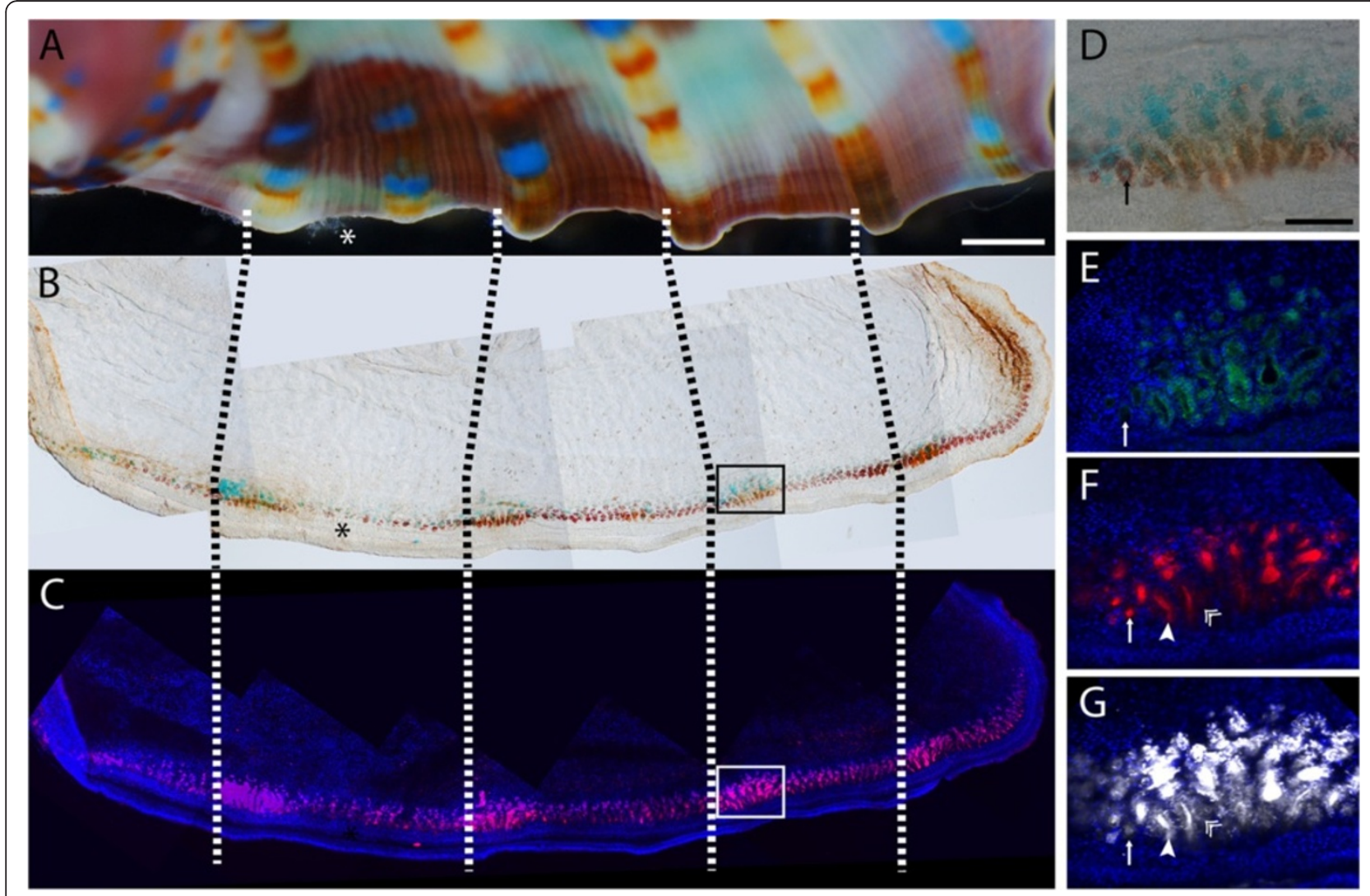

Figure 3 Pigmentation of the mantle corresponds to shell colour. A. Margin of the right side of a juvenile shell. Scale bar =0.5 mm. B. Mantle tissue directly underlying the shell margin depicted in $\mathbf{A}$. A line of pigmentation is visible, with colours generally corresponding to the colour of the shell in that region (dotted lines in panels $\mathbf{A}, \mathbf{B}$ and $\mathbf{C}$ align shell and mantle tissue). Areas with less visible pigment in the mantle correspond to white shell patches $(*)$. C. Confocal imaging of endogenous mantle fluorescence. Pigments fluoresce in green (not visible due to low intensity), red and far-red wavelengths. Cell nuclei have been stained with DAPI (blue fluorescence). D. Higher magnification of the boxed area in B. Blue, red and orange pigment can be observed. Often, more than one pigment can be seen in a single tubule (black arrow). Scale bar $=0.1 \mathrm{~mm}$. E, F, G. Confocal imaging of autofluorescent pigments in D. E. Excitation at $433 \mathrm{~nm}$ produces green autofluorescence, overlapping with orange pigment in $\mathbf{D}$. F. Excitation at $543 \mathrm{~nm}$ produces red autofluorescence, overlapping with blue pigment in D. G. Excitation at $633 \mathrm{~nm}$ produces far-red autofluorescence, also overlapping with blue pigment in $\mathbf{D}$. Red pigment in $\mathbf{D}$ produces no detectable autofluorescence (arrows in $\mathbf{D}, \mathbf{E}, \mathbf{F}, \mathbf{G}$ ). Arrowheads indicate fluorescent material within the lumen, double arrowheads indicate punctate fluorescent material that is likely intracellular. 

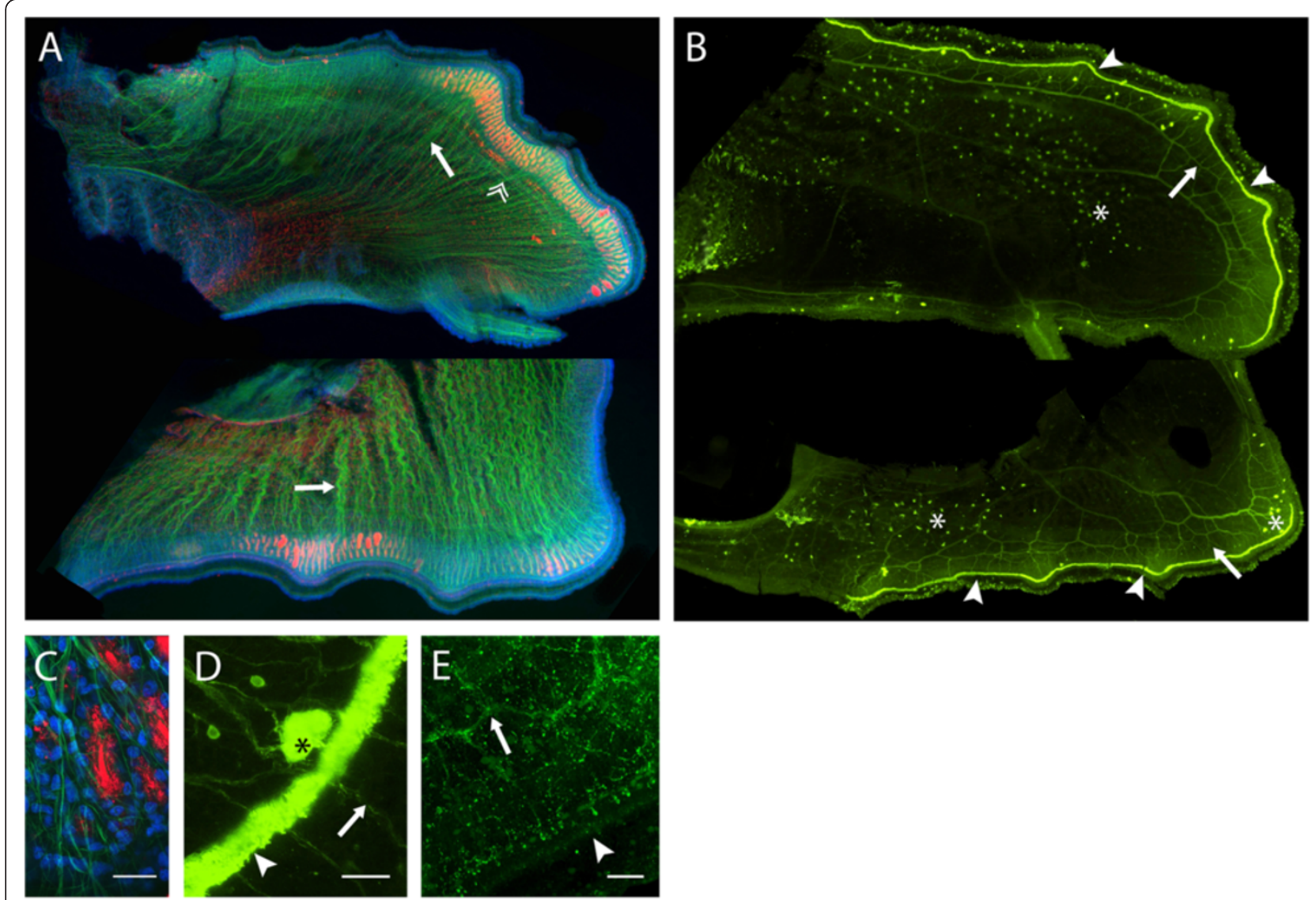

Figure 4 Musculature and innervation of the mantle. A. Localisation of actin filaments in the juvenile mantle revealed by phallacidin staining (green). Dorsal view of left (upper) and right (lower) mantle lobes, anterior is to the right. The majority of filaments lie perpendicular to the mantle edge (arrows), however some longitudinal fibres can be seen (double arrowhead). Pigment autofluorescence is visible within some tubules (red), nuclei are stained with DAPI (blue). B. Innervation of the juvenile mantle revealed by anti-acetylated tubulin immunoreactivity (green), orientation as in A. Nerve fibres become more dense in the distal edge of the mantle, in the proximity of the tubules and periostracal groove (arrows). Cilia within the groove are also labelled (arrowheads), as are individual ciliated cells $\left(^{*}\right)$. C. Higher magnification of A, showing muscle fibres (green) running between pigment-filled tubules (red). DAPI-stained cell nuclei are in blue. Scale bar $=15 \mu \mathrm{m}$. D. Higher magnification of B, showing nerve fibres extending past the tubular zone into the inner fold (arrow). Cilia within the periostracal groove (arrowhead) and a ciliated cell (*) are also visible. Scale bar $=10 \mu \mathrm{m}$. E. Distribution of the neuropeptide FMRF-amide revealed by immunostaining (green). Dorsal view of a similar region to $\mathbf{D}$, the opening to the periostracal groove is indicated by an arrowhead. Staining reveals increased complexity of neuronal structures than that visualised by tubulin staining (arrow). Scale bar $=30 \mu \mathrm{m}$. The bottom of the image is towards the mantle edge in $\mathbf{C}, \mathbf{D}$ and $\mathbf{E}$.

repositioning of the mantle by sensing the existing shell patterning is therefore unnecessary, at least in this species see [5]. This is also supported by the stripes of colour that can be seen in the shell, running parallel to the ridges (Figure 3A). These stripes likely represent the secretory product of a single tubule or set of tubules, and the colour change visible within a single stripe could reflect a change in pigment production within that particular tubule.

We propose that the pigment first accumulates in vesicles within the cells of the tubule, before being released into the lumen. This is supported by the similarity in appearance of secreted material and the material inside vesicles (Figure 2), as well as the confocal images in Figure $3 \mathrm{~F}$ and $\mathrm{G}$, which show intense streaks of fluorescence that are clearly within the lumen of the tubule, as well as fainter, more punctate staining surrounding the lumen, which is likely to reflect intracellular autofluorescent material. The presence of tubules in different states of secretion (empty, accumulating, or secreting) and the variation of pigment observed within the tubules of a single mantle suggests that pigment secretion operates in a modular fashion, i.e., that regulatory elements are acting on an entire tubule, rather than on individual cells. The regulatory mechanism for shell pigmentation is largely thought to be based upon reaction-diffusion events $[1,6,7]$, however it has also been proposed that the stimulus could be neural in nature [5,9]. As both neural and hormonal stimuli are known to induce vesicle release, we investigated innervation within this region of the mantle. 
Immunostaining with anti-acetylated tubulin reveals extensive innervation of the mantle, with the majority of nerve fibres terminating in the distal part of the inner fold (i.e., not near the tubules, Figure $4 \mathrm{~B}$ and D). However, immunostaining with an antibody directed against the FMRFamide neuropeptide shows a large amount of reactivity in the vicinity of the tubules (Figure $4 \mathrm{E}$ ). We were unable to determine if the tubules were directly innervated.

\section{Pigmentation in the abalone shell is localised to the prismatic layer}

$H$. asinina is the only mollusc to date for which the expression of a gene has been found to correlate with pigmentation patterns. This gene, Sometsuke, is expressed in regions of the mantle that directly underlie red shell patches $[19,28]$. The gene is expressed in the part of the mantle responsible for producing the prismatic layer, and appears to be present in structures that resemble tubules [26]. Based on this expression, and the localisation of pigment-containing tubules to the prismatic mantle zone, we hypothesised that pigment should be deposited within the prismatic shell layer in juvenile $H$. asinina.

Scanning electron microscopy (SEM) of a broken juvenile shell shows a clear distinction between prismatic (upper) and nacreous (lower) layers, with the prismatic layer having a width of approximately $30 \mu \mathrm{m}$ (Figure 5A). Light microscopy of a broken shell of a juvenile of comparable size clearly shows that the upper prismatic layer contains both red and blue pigments (Figure 5B). No pigmentation can be observed in the nacreous layer. Molluscan shells are typically covered by an outer proteinaceous layer, the periostracum, which is secreted from the periostracal groove (Figure 2A) and is often pigmented [16]. The periostracum in juvenile $H$. asinina is extremely thin (see in Additional file 2: Figure S2 for SEM of the shell and periostracum), and likely to not be coloured, given the lack of pigment in cells surrounding the periostracal groove.

It is probable that pigment production is not the only role of tubules. This region of the mantle is thought to be responsible for the secretion of the elements required to construct the prismatic layer, including organic matrix components and, possibly, calcium ions. The localisation of the matrix proteins MSI31 and MSI60 to similar tubular structures of the adult mantle of $H$. tuberculata [11] indicates that pigment and biomineralisation proteins are co-localised and likely co-produced.

\section{Distribution of tubules in other molluscs}

To determine whether tubules are restricted to abalone, we investigated mantle ultrastructure in a false abalone (Stomatella impertusa, Figure 6A) and a keyhole limpet (Diodora sp., Figure 6B). The morphology of the false
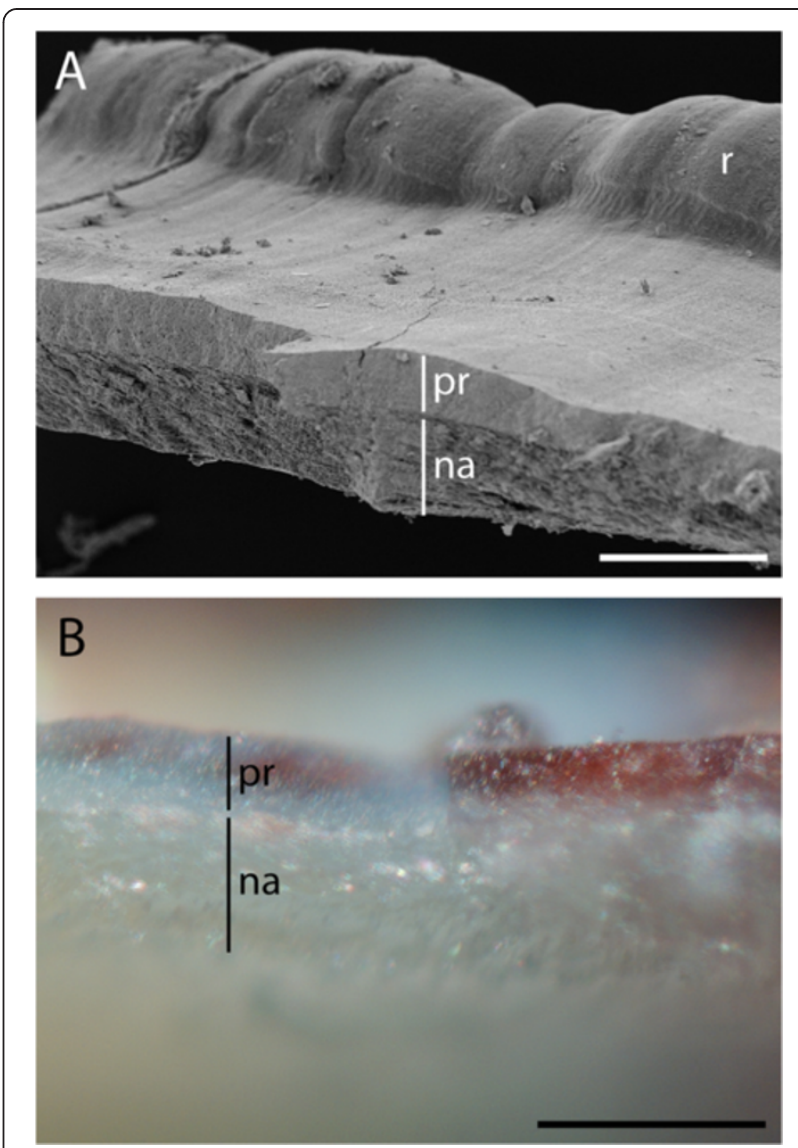

Figure 5 Pigment is localised to the prismatic layer. Scale bars $=200 \mu \mathrm{m}$. A. SEM of a broken fragment of juvenile shell. A shell ridge $(r)$ is visible. The two main shell layers, the prismatic layer (pr) and the nacreous layer (na) are clearly discernable. B. Light microscopy of a similar shell fragment. Pigment is clearly localised to the upper prismatic layer (pr), while the nacreous layer (na) is unpigmented.

abalone mantle was similar to that of $H$. asinina (Figure $6 \mathrm{C}$ ), however tubules were not visible in toluidine blue stained sections. Despite this, TEM revealed the presence of microvilli-lined invaginations in a similar region of the mantle in which tubules are found in $H$. asinina (Figure 6D). These invaginations appear more convoluted and less regular than abalone tubules, as are the cells lining the invaginations. These appear to contain fewer vesicles (compare Figure 6E to Figure $2 \mathrm{~B}$ and $\mathrm{C}$ ), although secreted material could be found in the lumen of the invagination. The mantle of the keyhole limpet is very different, consisting of three mantle folds (Figure 6F). While small infoldings could be found in the epithelium, these were associated with small papillae-like structures (Figure 6G), and were clearly distinct from the larger invaginations observed in $H$. asinina or the false abalone. We conclude that tubules are not restricted to abalone, but are also not universal in gastropod species. Numerous ultrastructural studies have been performed on bivalve mantle (e.g., [13,29,30]), however, to our knowledge, 

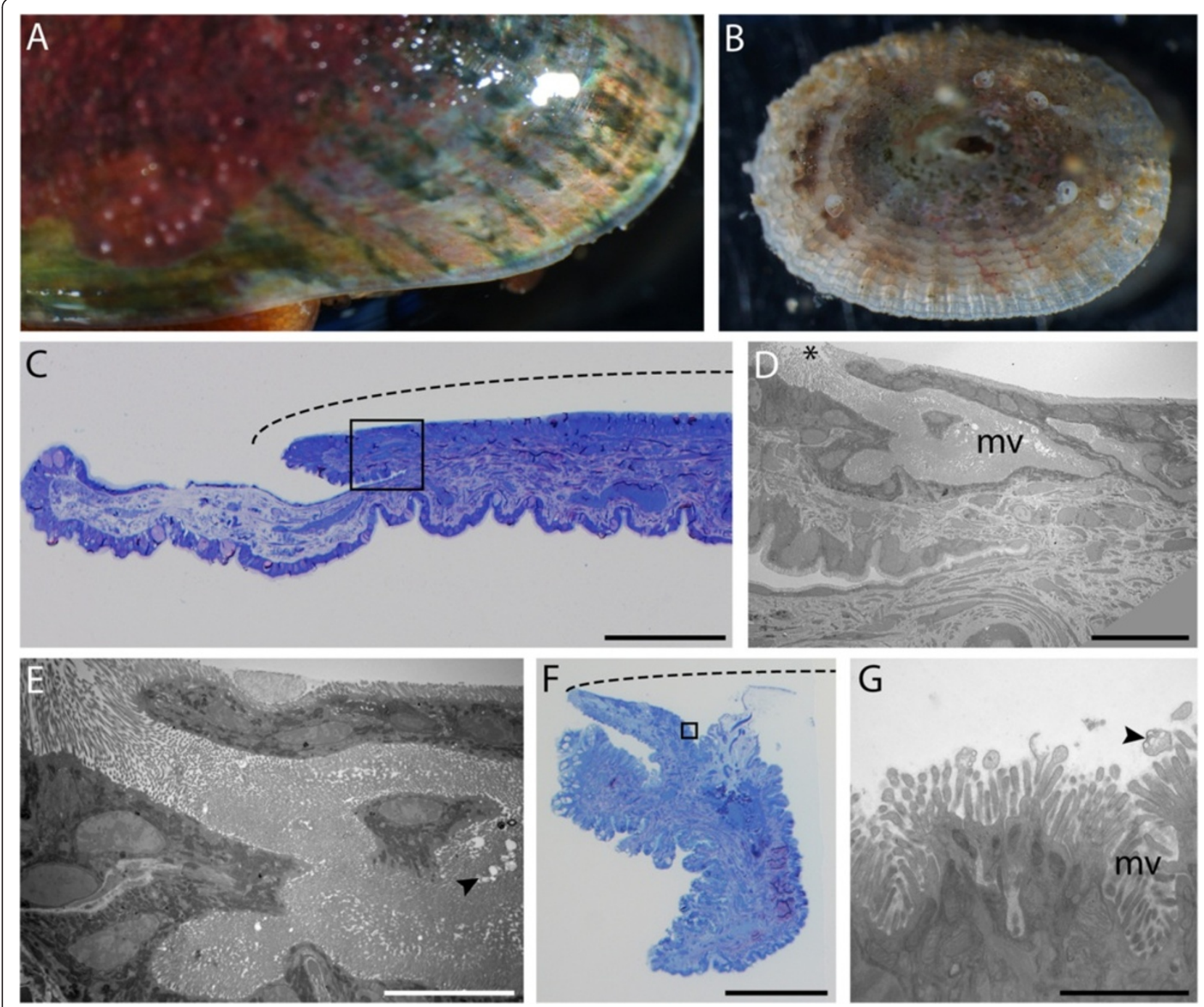

Figure 6 Presence or absence of tubules in other gastropod species. A. Shell of the false abalone Stomatella impertusa. B. Shell of the limpet Diodora sp. C. Toluidine blue stained cross-section of the mantle of the false abalone in A. The location of the shell is indicated by a dotted line. Scale bar $=100 \mu \mathrm{m}$. D. TEM of boxed area in C. Several tubule-like invaginations are visible. These are filled with microvilli (mv), and open to the dorsal surface $\left(^{*}\right)$. Scale bar $=20 \mu \mathrm{m}$. E. Higher magnification of $\mathbf{D}$. The cells lining the invagination are not as columnar as those lining abalone tubules (compare to Figure 2B and C), and do not appear to contain electron-lucent vesicles. Despite this, secretions can be observed within the lumen (black arrowhead). Scale bar $=10 \mu \mathrm{m}$. $\mathbf{F}$. Toluidine blue stained cross-section of the mantle of the keyhole limpet in $\mathbf{B}$. The location of the shell is indicated by a dotted line. Scale bar $=100 \mu \mathrm{m}$. G. TEM of boxed area in F. Some folding of the epithelium is apparent, but these are no deeper than one cell layer. The epithelium is covered with microvilli (mv), and secretions are visible (black arrowhead). Scale bar $=2 \mu \mathrm{m}$.

tubular structures have not been reported. We also acknowledge that tubules may not be present throughout all post-larval stages [12].

\section{Conclusions}

In this study we find that, in the juvenile abalone, shell colouration is controlled by the secretion of pigments from tubular structures localised within a region of the mantle that gives rise to the prismatic shell layer. We also find that similar structures can be found in other gastropod species. These findings reveal that pigmentation is controlled not by individual cells, but by numerous cells that secrete products into a common duct, and that the cellular basis of shell pigmentation is more complex than previously appreciated. A complete understanding of shell patterning mechanisms will require identification of the inductive trigger, unravelling of underlying interactions, and the source and identity of the various pigments that are observed.

\section{Methods}

Animal collection and husbandry

Spawning and larval culture of juvenile $H$. asinina was carried out at Heron Island Research Station according to 
the methods previously described [23]. Upon competency (96-108 hours post fertilisation), larvae were settled in a tank containing biofilmed perspex sheets and the articulated coralline algae Amphiroa spp. [31]. After 8 hours, a flow of seawater was provided. Juvenile $H$. asinina were reared in this tank for 2-4 months prior to transportation to the aquarium facilities in the School of Biological Sciences at the University of Queensland. Juveniles fed on algae naturally growing within the tanks.

\section{Fixation}

Prior to fixation animals were relaxed in $1 \mathrm{M} \mathrm{MgCl}_{2}$ in filtered seawater. Fixation for electron microscopy followed the method of Eisenman and Alfert [32].

\section{Electron microscopy}

Samples were dehydrated in an ethanol series and embedded in EPON 812 resin. Sections were cut at $60 \mathrm{~nm}$ using a Leica UC6 ultramicrotome, contrasted with uranyl acetate and lead citrate and viewed in a JEOL JEM 1011 transmission electron microscope at $80 \mathrm{kV}$. Samples for SEM were mounted on stubs and sputter-coated with gold before viewing in a JEOL JCM 5000 Neoscope at $10 \mathrm{kV}$. Mantle tissue for 3View analysis was processed according to previously described methods [33]. Samples were imaged in a Zeiss Sigma SEM fitted with a Gatan 3 View 2XP system. Chamber pressure was $10 \mathrm{~Pa}$ and the accelerating voltage was $2.2 \mathrm{kV}$. The z-step size was $50 \mathrm{~nm}$ and samples were imaged at a magnification of $5000 \times$.

\section{Pigmentation mapping}

Juvenile abalone were anesthetised in $1 \mathrm{M} \mathrm{MgCl}_{2}$ in filtered seawater for $10 \mathrm{~min}$ and shells were photographed using a Nikon Digital Sight DS-U1 camera on an Olympus SZX7 dissecting microscope. Mantle tissue was dissected and mounted on slides in Prolong Gold anti-fade reagent (Invitrogen, P36930) containing $5 \mathrm{ng} / \mathrm{mL}$ DAPI Nucleic Acid Stain (Invitrogen, D1306). Light microscopy was performed on an Olympus BX60 compound microscope with Nomarski optics. Confocal imaging was performed using a Zeiss LSM 510 META Confocal Microscope. In order to capture the entire mantle, multiple confocal (single plane) and light microscopy images were taken using the same settings for each individual, and were stitched together manually in Adobe Photoshop CS2. Any brightness or contrast adjustments were applied to the entire image after stitching.

\section{Histology and Immunohistochemistry}

Sectioning and toluidine blue staining were performed as previously described [10].

For phallacidin staining, fixed juveniles stored in PBTw ( $1 \times$ PBS, $0.1 \%$ Tween 20 ) were decalcified by incubation in $1 \times$ PBS, $4 \%$ paraformaldehyde and $350 \mathrm{mM}$ EDTA for $2 \mathrm{~h}$, after which the remaining organic shell remnants were dissected away. Samples were permeabilised in $0.2 \%$ PBTx (1× PBS, $0.2 \%$ Triton X) for $1 \mathrm{~h}$ at room temperature (RT) prior to incubation in a 1:40 dilution (of $6.6 \mu \mathrm{M}$ stock) BODIPY FL-Phallicidin (Invitrogen, B607) for $1 \mathrm{~h}$ at RT, and then washed twice in 1x PBS.

For immunohistochemistry, juveniles were decalcified as described above and then permeabilised by four 30 min washes in $0.2 \%$ PBTx. Blocking was performed in $1 \times$ PBS, $0.1 \%$ Triton X and $0.2 \%$ BSA for $2 \mathrm{~h}$. Samples were then incubated in a 1:500 dilution of Anti-Acetylated Tubulin (Sigma, T7451, mouse) or a 1:250 dilution of Anti-FMRFamide (Abcam, ab10352, rabbit) in blocking solution for $12-24 \mathrm{~h}$ at $4^{\circ} \mathrm{C}$. Four $30 \mathrm{~min}$ washes in $0.1 \%$ PBTx were then performed prior to incubation in secondary antibodies (Alexa Fluor 488-conjugated goat anti-mouse, Invitrogen A11001, or Alexa Fluor 488conjugated donkey anti-rabbit, Invitrogen A21206) for $12-24 \mathrm{~h}$ at $4^{\circ} \mathrm{C}$. This was followed by another four 30 min washes in $0.1 \%$ PBTx.

Stained mantle tissue was dissected and mounted as above.

\section{Additional files}

Additional file 1: Movie S1. Serial blockface imaging taken using Gatan 3View showing several tubules. Tubules contain differing amounts of secreted products, and two tubules are seen to merge.

Additional file 2: Figure S1. Additional examples of congruence between shell patterning and mantle pigmentation. Figure S2. SEM of the juvenile $H$. asinina shell, showing the thin periostracum.

\section{Abbreviations}

TEM: Transmission electron microscopy; SEM: Scanning electron microscopy.

\section{Competing interests}

The authors declare that they have no competing interests.

\section{Authors' contributions}

$A B$ performed immunostaining, histology, and confocal microscopy. CM performed light and confocal microscopy. KG performed histology, electron microscopy, and 3View analyses. CM, AB and BD conceived of the study, participated in the design, and drafted the manuscript. All authors read and approved the final manuscript.

\section{Acknowledgements}

The authors thank staff at Heron Island Research Station for their assistance in maintaining juvenile abalone, Ms Robyn Webb for assistance with collecting the 3View data and Dr Stephen Mason (UQ) for assistance with confocal microscopy. The authors acknowledge the facilities and the scientific and technical assistance of the Australian Microscopy and Microanalysis Research Facility at the Centre for Microscopy and Microanalysis, The University of Queensland. This research was supported by funding from the Australian Research Council to BMD.

\section{Author details}

${ }^{1}$ Centre for Marine Science, School of Biological Sciences, The University of Queensland, Brisbane, QLD 4072, Australia. ${ }^{2}$ Centre for Microscopy and Microanalysis, The University of Queensland, Brisbane, QLD 4072, Australia. 
Received: 4 June 2014 Accepted: 15 August 2014

Published online: 02 September 2014

\section{References}

1. Waddington $\mathrm{CH}$, Cowe RJ: Computer simulation of a mulluscan (sic) pigmentation pattern. J Theor Biol 1969, 25:219-225.

2. Tabata T, Takei Y: Morphogens, their identification and regulation. Development 2004, 131:703-712.

3. Lindsay D: Simulating molluscan shell pigment lines and states: implications for pattern diversity. Veliger 1982, 24:297-299.

4. Lindsay DT: A new programmatic basis for shell pigment patterns in the bivalve mollusc Lioconcha castrensis (L.). Differentiation 1982, 21:32-36.

5. Boettiger A, Ermentrout B, Oster G: The neural origins of shell structure and pattern in aquatic mollusks. Proc Natl Acad Sci U S A 2009, 106:6837-6842.

6. Kusch I, Markus M: Mollusc shell pigmentation: cellular automaton simulations and evidence for undecidability. J Theor Biol 1996, 178:333-340.

7. Meinhardt $\mathrm{H}$ : Models for positional signalling, the threefold subdivision of segments and the pigmentation pattern of molluscs. J Embryol exp Morph 1984, 83(Supplement):289-311.

8. Meinhardt $\mathrm{H}$, Klingler $\mathrm{M}$ : A model for pattern formation on the shells of molluscs. J Theor Biol 1987, 126:63-89.

9. Ermentrout B, Campbell J, Oster G: A model for shell patterns based on neural activity. Veliger 1986, 28:369-388.

10. McDougall C, Green K, Jackson DJ, Degnan BM: Ultrastructure of the mantle of the gastropod Haliotis asinina and mechanisms of shell regionalization. Cells Tissues Organs 2011, 194:103-107.

11. Jolly C, Berland S, Milet C, Borzeix S, Lopez E, Doumenc D: Zonal localization of shell matrix proteins in mantle of Haliotis tuberculata (Mollusca, Gastropoda). Mar Biotechnol 2004, 6:541-551.

12. Sud D, Poncet J, Saihi A, Lebel J, Doumenc D, Boucaud-Camou E: A cytological study of the mantle edge of Haliotis tuberculata L. (Mollusca, Gastropoda) in relation to shell structure. J Shellfish Res 2002, 21:201-210.

13. Beedham G: Observations on the mantle of the Lamellibranchia. Quart $J$ Mic Sci 1958, 3:181-197.

14. Lowenstam HA, Weiner S: On Biomineralization. New York: Oxford University Press; 1989.

15. Comfort A: The pigmentation of molluscan shells. Biol Rev 1951, 26:285-301.

16. Needham A: The zoochromes of helicid shells. Naturwissenschaften 1975, 62:183-184

17. Li X, Bai Z, Luo H, Liu Y, Wang G, Li J: Cloning, differential tissue expression of a novel hcApo gene, and its correlation with total carotenoid content in purple and white inner-shell color pearl mussel Hyriopsis cumingii. Gene 2014, 538:258-265.

18. Gantsevich M, Tyunnikova A, Malakhov V: The genetics of shell pigmentation of the Mediterranean mussel Mytilus galloprovincialis Lamarck, 1819 (Bivalvia, Mytilida). Dokl Biol Sci 2005, 404:370-371.

19. Jackson DJ, McDougall C, Green K, Simpson F, Wörheide G, Degnan BM: A rapidly evolving secretome builds and patterns a sea shell. BMC Biol 2006, 4:40.

20. Jackson DJ, McDougall C, Woodcroft B, Moase P, Rose RA, Kube M, Reinhardt R, Rokhsar DS, Montagnani C, Joubert C, Piquemal D, Degnan BM: Parallel evolution of nacre building gene sets in molluscs. Mol Biol Evol 2010, 27:591-608.

21. Marie B, Marie A, Jackson DJ, Dubost L, Degnan BM, Milet C, Marin F: Proteomic analysis of the organic matrix of the abalone Haliotis asinina calcified shell. Proteome Sci 2010, 8:54.

22. Counihan RT, McNamara DC, Souter DC, Jebreen EJ, Preston NP, Johnson CR, Degnan BM: Pattern, synchrony and predictability of spawning of the tropical abalone Haliotis asinina from Heron Reef, Australia. Mar Ecol Prog Ser 2001, 213:193-202.

23. Jackson DJ, Ellemor N, Degnan BM: Correlating gene expression with larval competence, and the effect of age and parentage on metamorphosis in the tropical abalone Haliotis asinina. Mar Biol 2005, 147:681-697.

24. Crofts DR: Haliotis. $L M B C$ memoirs on typical British marine plants and animals 1929, 29:1-174.

25. Emberton L: Relationships between pigmentation of shell and of mantle in the snails Cepaea nemoralis (L.) and Cepaea hortensis (Mull.). Proc Zool Soc Lond 1963, 140:273-293.
26. Checa AG, Salas C, Harper EM, Bueno-Pérez JD: Early stage biomineralization in the periostracum of the 'living fossil' bivalve Neotrigonia. PLoS One 2014, 9:e90033.

27. Standring S: Gray's Anatomy: the anatomical basis of clinical practice. 40th edition. Edinburgh: Churchill Livingstone: Elsevier; 2008.

28. Jackson DJ, Wörheide G, Degnan BM: Dynamic expression of ancient and novel molluscan shell genes during ecological transitions. BMC Evol Biol 2007, 7:160.

29. Jabbour-Zahab R, Chagot D, Blanc F, Grizel H: Mantle histology, histochemistry and ultrastructure of the pearl oyster Pinctada margaritifera (L.). Aquat Living Resour 1992, 5:287-298.

30. Colville A, Lim R: Microscopic structure of the mantle and palps in the freshwater mussels Velesunio ambiguus and Hyridella depressa (Bivalvia: Hyriidae). Molluscan Res 2003, 23:1-20.

31. Williams EA, Craigie A, Yeates A, Degnan SM: Articulated coralline algae of the genus Amphiroa are highly effective natural inducers of settlement in the tropical abalone Haliotis asinina. Biol Bull 2008, 215:98-107.

32. Eisenman $\mathrm{E}$, Alfert $\mathrm{M}$ : A new fixation procedure for preserving the ultrastructure of marine invertebrate tissues. J Microsc 1982, 125:117-120.

33. Wilke SA, Antonios JK, Bushong EA, Badkoobehi A, Malek E, Hwang M, Terada M, Ellisman $\mathrm{MH}$, Ghosh A: Deconstructing complexity: serial block-face electron microscopic analysis of the hippocampal mossy fiber synapse. J Neurosci 2013, 33:507-522.

doi:10.1186/s12983-014-0062-0

Cite this article as: Budd et al:: Control of shell pigmentation by secretory tubules in the abalone mantle. Frontiers in Zoology 2014 11:62.

\section{Submit your next manuscript to BioMed Central and take full advantage of:}

- Convenient online submission

- Thorough peer review

- No space constraints or color figure charges

- Immediate publication on acceptance

- Inclusion in PubMed, CAS, Scopus and Google Scholar

- Research which is freely available for redistribution 